\title{
Energy Efficient Strategy for WSN Technology Using Modified HGAF Technique
}

\author{
https://doi.org/10.3991/ijoe.v17i06.17739 \\ Hanane Aznaoui ${ }^{(凶)}$, Said Raghay \\ Cadi Ayyad University, Marrakech, Morocco \\ H. aznaoui@gmail.com \\ Arif Ullah, Mubashir Hayat Khan \\ Universiti Tun Hussein Onn Malaysia, Parit Raja, Malaysia
}

\begin{abstract}
Due to the rapid growth in technologies and services, the development of sensor nodes has imposed great challenges for researchers in improving our daily life in all domains such as environmental research, health care, monitoring, military, and physical activity recording. A wireless sensor network (WSN) is therefore a collection of a small and large number of sensor nodes able of collecting, communicating with each other, and transmitting the collected data to a base station. For analyzing the performance of a WSN, different algorithms were developed and used, and the Hierarchical Geographic Adaptive Fidelity (HGAF) is one of the most popular energy-efficient routing protocols. The decrease in energy use in the wireless sensor network directly affects the life of the network. HGAF is one of the important multiple locations based on the routing system algorithm, its main function technique is to deactivate unnecessary nodes from the network without interrupting the other connected nodes. In this article, we have proposed a technique known as Modified Geographic Adaptive Fidelity (M.HGAF) protocol and its design as a power-saving meth-od. In our proposed technique, the size of the cell structure of the grid has changed and the communication method improves due to these changes. According to our results, the proposed technique increased the dead node rate by about $25 \%$, which improved the network lifetime.
\end{abstract}

Keywords - Energy Aware, Data Communication, Routing System, Wireless Sensor Network

\section{$1 \quad$ Introduction}

Wireless Sensor Network (WSN) is a combination of various sensors with a specific memory, power, transmitter, and receiver. Its process of data collection is carried out from the neighboring networks, then transmitting the collected data to an integrated location. WSN consists of a large number of multifunctional sensor nodes used for collecting and sending data in different environments [1,2]. These sensor nodes typically have the ability of sensing, processing, and transmitting monitored data to the 
base station (BS) or the sink node in a direct or multi-hop transmission manner [3,4]. At a practical level, the sensors are used in control systems in various applications and fields including industry, transport, healthcare, science, and environment monitoring [5]. In a WSN, each node has one or more sensors, integrated processors as well as low-power radios which are battery operated. One of the main roles of WSN is its deployment of hundreds of sensors in real-time environments [6,7]. Regarding the environments and user demands, the WSNs are classified into different categories: multimedia sensor network, mobile sensor network, body area sensor networks, and underwater wireless sensor.

Nowadays, WSNs attract more researchers because of the ubiquity and their tremendous development in the internet of things (IoT). Although networks provide various advantages, WSN technology still faces different types of challenges such as energy efficiency, complexity, scalability, delay, and robustness [8,9]. Figure 1 presents the structure of WSN.

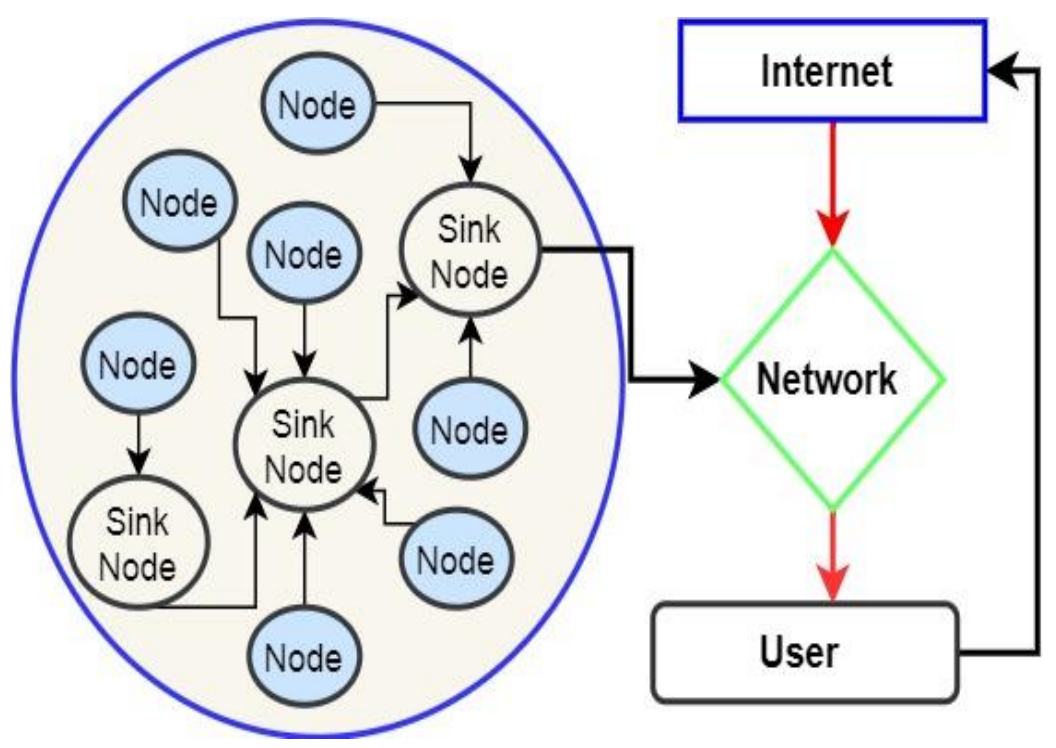

Fig. 1. Structure of WSN

Currently, sensors are not only used for data connection, but also for bidirectional purpose, which means that other activities are controlled by the central system. In some situations, the sensor is made up of batteries that cannot be changed or recharged by the system, which affects the performance of the system. In this context, different protocols have been used and adopted in WSN technology. In this paper, we focused on hierarchical based routing protocols. This kind of routing used a technique in which some node has more energy than other nodes and these nodes are used for the communication process. The hierarchical systems also use a clustering system of nodes and in this situation; the nodes make cluster groups where each node joined a 
cluster. This clustering scheme is more energy-efficient and easily for the manager $[10,11]$.

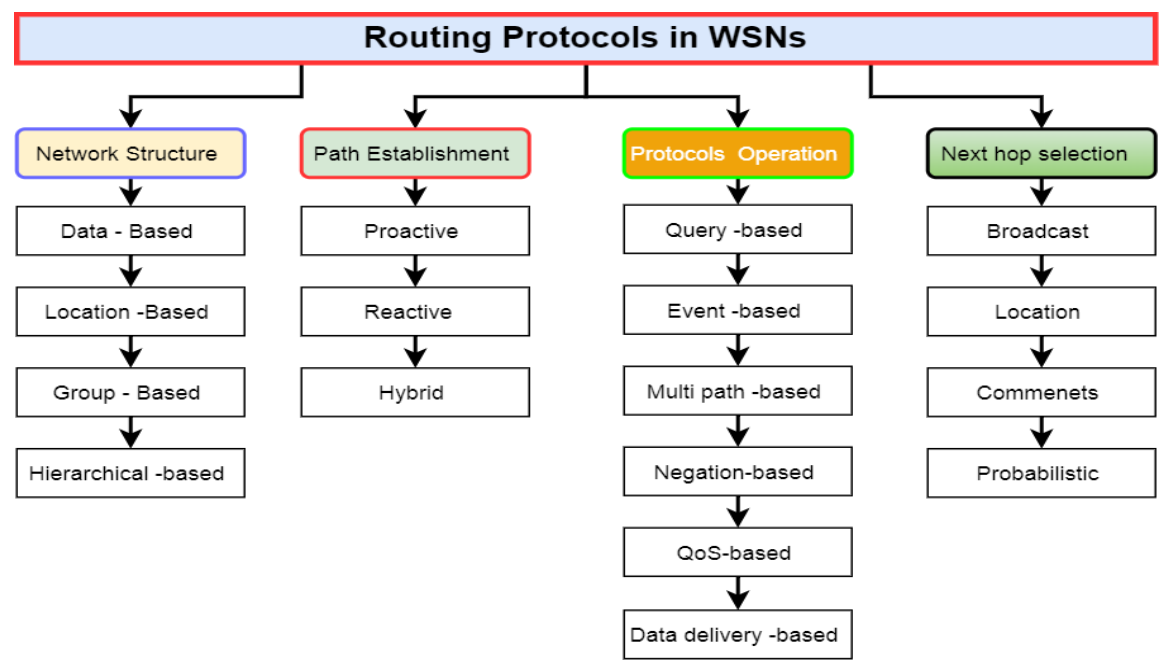

Fig. 2. Type of protocols used in WSN

The active node selection process is the selecting of an active node from those entire nodes which are present in the sub cell. In sub-sell selection methods, all nodes of the sub cells having high energy levels will be selected and the remaining will go into sleep mode.[12]. Figure 2 present the different categories of protocols which are used in WSN where we selected hierarchical based protocol known as Geographic Adaptive Fidelity. For the comfort of readers, we provided a list of the most frequently used acronyms in the paper are mention in table 1.

Table 1. Acronyms List

\begin{tabular}{|l|l|l|l|}
\hline Acronyms & \multicolumn{1}{|c|}{ Meaning } & Acronyms & \multicolumn{1}{c|}{ Meaning } \\
\hline WSN & Wireless sensor network & GAF & Geographical adaptive fidelity \\
\hline IoT & Internet of thing & HGAF & Hierarchical geographic adaptive fidelity \\
\hline Td & Predefine time & CEC & Cluster-based energy conservation \\
\hline SG & Smart grid & BS & Base station \\
\hline
\end{tabular}

\section{Related Work}

Different routing protocols are used to solve the energy issue or improve the network lifetime system in WSNs. To ensure these improvements, several and different algorithms and methods are used. Normally two main algorithms are adopted are distinctive and classification technique. Energy proficient routing protocols are used to increase the network lifetime or node lifetime and geographic adaptive fidelity (GAF) is one of them [13]. In WSNs, routing protocols are divided according to the 
network structure like flat-based, clustering-based, hierarchical based and geographical routing. To increase the energy efficiency most of the routing protocols used a subset of sensor systems within the sensing region [14]. The energy management system is the main element that directly operational on network life. Regarding this, the authors proposed two main protocols which are Geographic Adaptive Fidelity (GAF) and Cluster-based Energy Conservation (CEC). The working of GAF is to identify the unneeded node, make off this kind of node ratio in the system, and make the radio range of a conservative system. However, CEC protocols are used to identify the duplication and redundancy system in the network to improve the energy system in WSN technology [15].

Based on the literature, these proposed technologies improve the network life system but it takes more time as compared to other techniques [16]. Energy consumption is one of the main challenges in WSN technology. In WSN technology two main types of nodes work, first of them collect and share information with different nodes; these use more energy. Second nodes forward this information to the base station. For improvements to these nodes system, the author's proposed protocols are known as modified Geographic Adaptive Fidelity (MGAF). These are location-based routing protocols that used these nodes based on the location system. The modification took place at turning off the system of the technique due to that section, it off those nodes which work the same functionalities, so for that reason, it improves the network lifetime [17]. Energy awareness is one of the main factors for the design of protocols and algorithms in WSN technology. Energy awareness system can be achieved through a different step which are node mobility, scalable routing, path duration, and reduce route discovery system. For improvement in energy systems, the researchers designed techniques based on routing strategy; and among the proposed techniques, there are Geographic Adaptive Fidelity (GAF) and Geographic and Energy Aware Routing (GEAR). The implementation of these protocols requires making a decision based on the location information instead of link information for routing. This technique is also known as position-based routing and these decide on those nodes which are sleeping state. Due to sleeping status, the technique improves the network lifetime as compared to other techniques [18]. WSN is active and popular in the research field due to its routing position in a different technique. GAF consists of three transition states which are discovery, sleeping, and active stage. Authors [19], improved this protocol based on its basic version. The proposed technique is known as EEGAF which improves the discovery status of the system and also optimizes the data sending. Due to these changes, the proposed technique improves the energy of the network due to which the network life improves. Based on the result of the proposed technique improves the network lifetime. WSN known by their sensor nodes and these nodes are directly connected to the base station to share the collected information.

\section{HGAF Algorithm and Working Steps}

HGAF is an energy-aware algorithm and design for the optimization process in WSN technology. This protocol works on the base of a Global Positioning System 
(GPS) system; it means that each node uses the location system along with forwarding packets in the network. GAF divided sensor nodes to equal to grid size because grid size is very important; if their size not equal then energy consumption affected the entire network. Therefore, it works properly and is based on a formula which is $\mathrm{r} \leq$ $\mathrm{R} / \sqrt{5}$ where $\mathrm{r} * \mathrm{r}$ presents the virtual grid and $\mathrm{R}$ presents the ratio of the grid system [20]. Adaptive geographic fidelity protocol is a location-based technique normally used for the energy-aware system. This routing protocol is used in the Adhoc network and WSNs. There are various issues exit in WSN but one of the main issues is save the energy of the node because when we deployed the sensor node, then it very difficult to replace or change the battery of the sensor node. The energy consumption makes the failure of the network or the node. For improvement in the energy system of the sensor node, different techniques are designed that improve the network life [21]. Figure 3 shows the steps used in the HGAF protocol. HGAF transition states consist of three main states which are sleeping state, discovery state, and active state and each state has its working criteria. The most proper state of the network is that only one node per grid will be active and other nodes will be in sleeping mode. This process is done due to saving energy, which each active and working node for the communication process will be selected based on its energy level. When the network starts working, certain nodes are in the discovery process while other nodes are sleeping or in the active state which is responsible for reporting collected data to sink node or base station $[22,23]$.

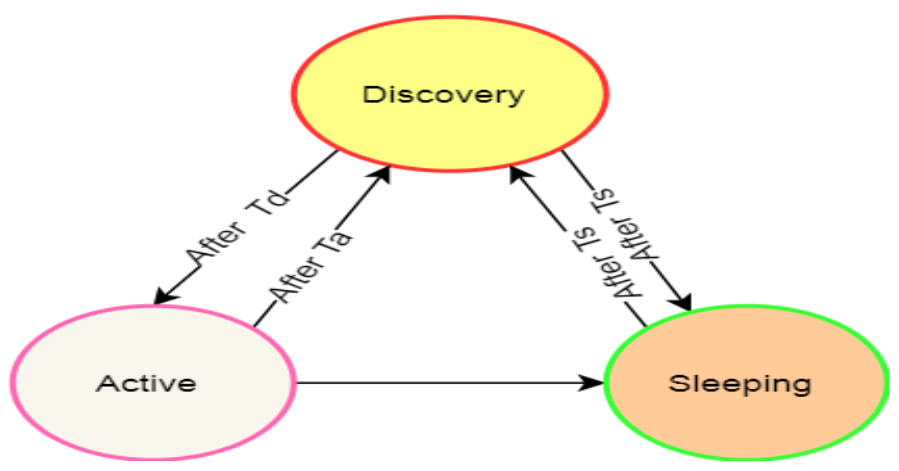

Fig. 3. Transition states of HGAF algorithm

As mention in figure 3, (i) Discovery to active state transition process: in this process, each node exchange message to each other, which are facing the equivalent grid. The message consists of node id, its current state, gird id, node state, and energy level. After the predefined time Td the node change to active level if not receive any other discovery message for the network [24, 25]; (ii) Active to sleeping transition process: in this process, the Ta shows the position of the node that how long node will be in the active state. After the Ta, if any other node finds which have a high level of energy in the same gird position then this node changes its position into sleeping mode; (iii) Sleeping to discovery state transition process [25,27]: when node completes it sleeping state time, Ts before wake up, it goes through the discovery phase. When the 
node again enters into a discovery phase, its network checks its energy level, if the energy level is high, the node goes to an active state, if not, it re-entire into sleeping mode [28.29]. Figure 4 shows the discovery process.

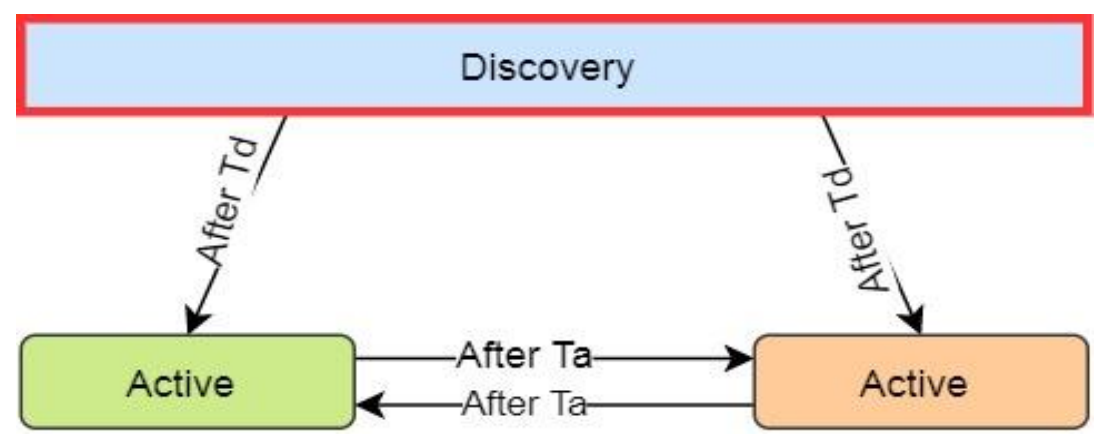

Fig. 4. Discovery process of HGWF algorithm

Active to discovery transition process, when a node enters into discovery mode, it broadcast the discovery message in the network in the same grid (Td). If it finds a message from a high energy level node, it enters in sleeping mode, or after Td time, it starts the same process again [30][31].

\section{Problems Statement}

For improvement of energy management system in WSNs technology, several types of research modify and enhance HGAF technique. As the study of related work, it seems that different research modified different sections of the algorithm and these all modifications took place for improvement in a network lifetime. The standard size of the cell is $\left(R^{2 / 2}\right)$ for connectivity, this standard size makes more active node in the region which make more energy usage in the network. Therefore, in this paper, we increase the cell connectivity size $\left(R^{2 / 3}\right)$ to increase the active node number used as a cluster head system.

\section{Proposed Modified HGAF Based Routing Protocol}

In the proposed technique the node selection method is used known as the hierarchical selection process. In which sub cell selection method, all nodes in sub cell having energy level high active position and remain will goes to sleep mode. In the communication of M.HGAF selection between two adjacent cells among node and active node. The distance between two extreme nodes in two adjacent cells $\left(f_{t}\right)$ and less communication range $r$ now it is time to decreasing the number of active nodes in the subcell where $N$ is present cell and sub-cell will be $n$. Figure 5 shows the working structure of M.HGAF in the grid. 


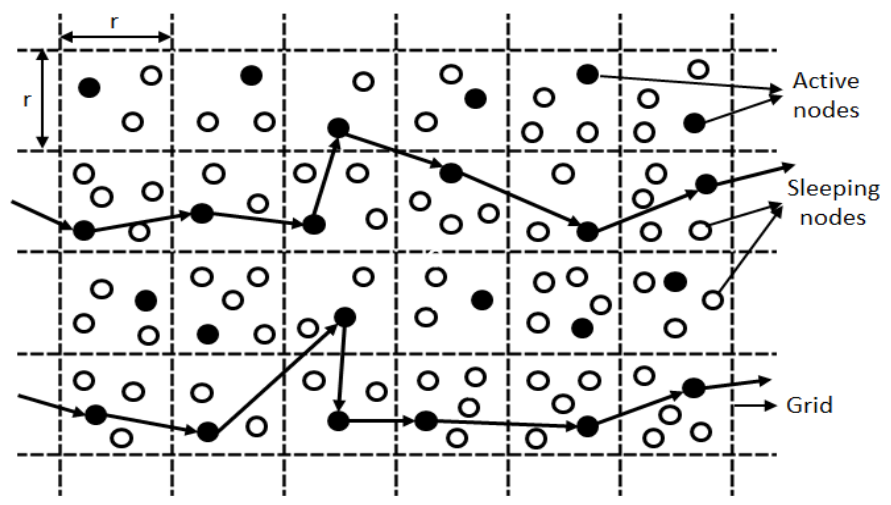

Fig. 5. Show the structure of HGAF working

Now we assume each cell is square with (Et) unit and each side along with sub cell is square with (Eo) To satisfy (Ft) the following condition will be checked

$$
\left.(O P)^{2}=(D n)^{2}+(N+1) d N\right)^{2} \leq R^{2}
$$

Where we can write the equations as

$$
F_{t} \leq \frac{1}{\sqrt{(N+1)^{2}+(1)}} R
$$

Let's use the length of the side of the cell $\left(D_{n)}=N_{d n}\right.$ to rewrite inequality

$$
D_{n} \leq \frac{N}{\sqrt{(N+1)^{2}+1}} R
$$

Thus, the cell area $S^{N}, S^{n}$ and area satisfies the following condition.

$$
\begin{aligned}
& s N=(d N)^{2} \leq \frac{1}{(N+1)^{2}+1} R^{2} \\
& S n=(D n)^{2} \leq \frac{N^{2}}{(N+1)^{2}+1} R^{2}
\end{aligned}
$$

The size of the cell can measure or communication range. As we know that in HGAF the active node acts as a cluster node and the active node in the same cell. The size must be a communication range will be. Figure 6 shows the activate process of sub cell in the grid of HGAF.

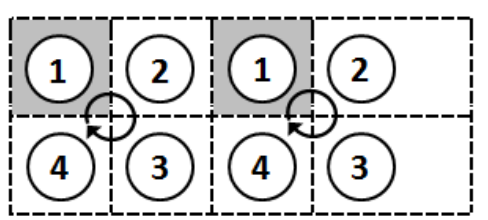

Active sub cell process

Fig. 6. Active process of sub cell in grid 


$$
\sqrt{2 D_{n}} \leq R
$$

The above equation further explain by presenting with equation (5) and (6)

$$
\frac{\sqrt{2 D}}{\sqrt{\left((N+1)^{2} \leq 1\right.}}
$$

The above equation shows the cell size when it $N \geq 3$ it means it enlarge and when it become $N \geq 2$ it means the size is ok. To find out subcell distance for communication. When the size of $\mathrm{N}$ in the old number than for the center point of sub cell as shown in figure 7.

$$
O W=O V+V M=\frac{\sqrt{2}}{2} D N+\frac{\sqrt{2}}{2} D N \leq R A
$$

Where $O W$ is the maximum distance of the active and other nodes. $R$ is the connectivity between the active and another node. $\mathrm{N}$ is the old number by replacing $\mathrm{N}$ with $\mathrm{N}=2 \mathrm{n}-1(\mathrm{n}=1,2,3 \ldots . . \mathrm{n})$. OW has a satisfied condition by inculcation. To analysis between cell and sub-cell for the probability of node in sub cell and cell the average numbers of nodes in each cell when each cell divided N2 and sub-cell $\mathrm{Bn}$ is calculated as follows.
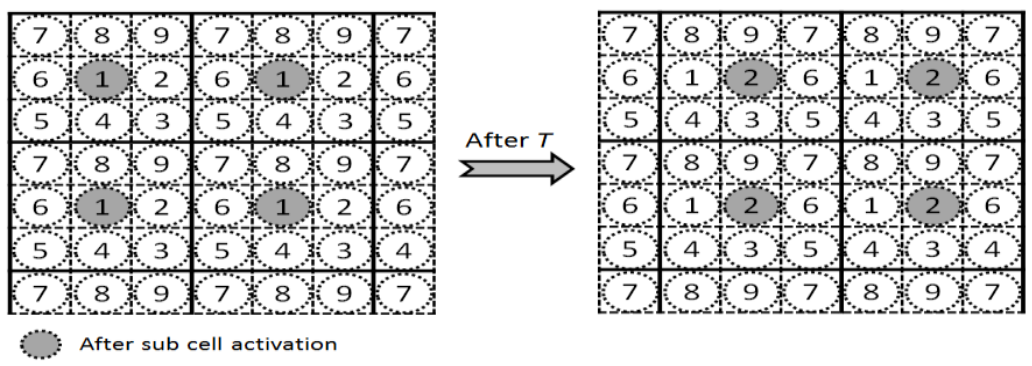

Fig. 7. After the active node selection in sub cell

$$
B_{N}=B \frac{S N}{S 1}=B_{1} \frac{5 N 2}{(N+1) 2}
$$

For sub-cell (1or $n 2$ ) $\mathrm{N}$ and B1 $\mathrm{S}$ is as follow

$$
\begin{aligned}
& P o(n)=\left(1-\frac{1}{N 2}\right) B^{N\left(11 \frac{I}{n 2}\right)} \\
&\left.\frac{5^{n 2} B 1}{1(N+1) 2}+1\right)
\end{aligned}
$$

After the section of sub cell, the next section is to find out the available sub-node selection and the equation number (11) shows the selection method. Node position error estimation rate. 


$$
\frac{N-1}{\sqrt{(N+!)^{2+1}}}
$$

After all the process now it time to estimate the error node of a dead node during the simulation process and equation 11 show the estimation rate in the network.

Simulations and results

For the verification of the proposed technique, the M.HGAF algorithm and their result were compared against its basic HGAF technique. While using main parameters to evaluate its performance. Table 2 shows the parameters that are used during the process.

Table 2. Parameter used for simulation process

\begin{tabular}{|l|c|l|c|}
\hline \multicolumn{1}{|c|}{ Parameter } & Value & \multicolumn{1}{c|}{ Parameter } & Value \\
\hline Network size & $100 \mathrm{M} \times 100 \mathrm{M}$ & EO & 0.25 \\
\hline Number of Grid cell & $3 \times 35 \times 57 \times 7$ & Eelec & $50 \mathrm{nj} / \mathrm{bit}$ \\
\hline Bs Coordinate & $50 \times 50$ & ETx & $50 \mathrm{nj} / \mathrm{bit}$ \\
\hline Sensor Node & 100 & Efs & $10 \mathrm{pj} / \mathrm{bit} / \mathrm{m} 2$ \\
\hline Transmission Range & $100 \mathrm{M}$ & Probability of optimal CH & 0.1 \\
\hline Routing Protocols & HGAF and M.HGAF & Probability of optimal Advance C node & 0.1 \\
\hline
\end{tabular}

In the simulation, we check the communication of the node in gird after changing its position figure 8 shows the two-algorithm communication in the grids.

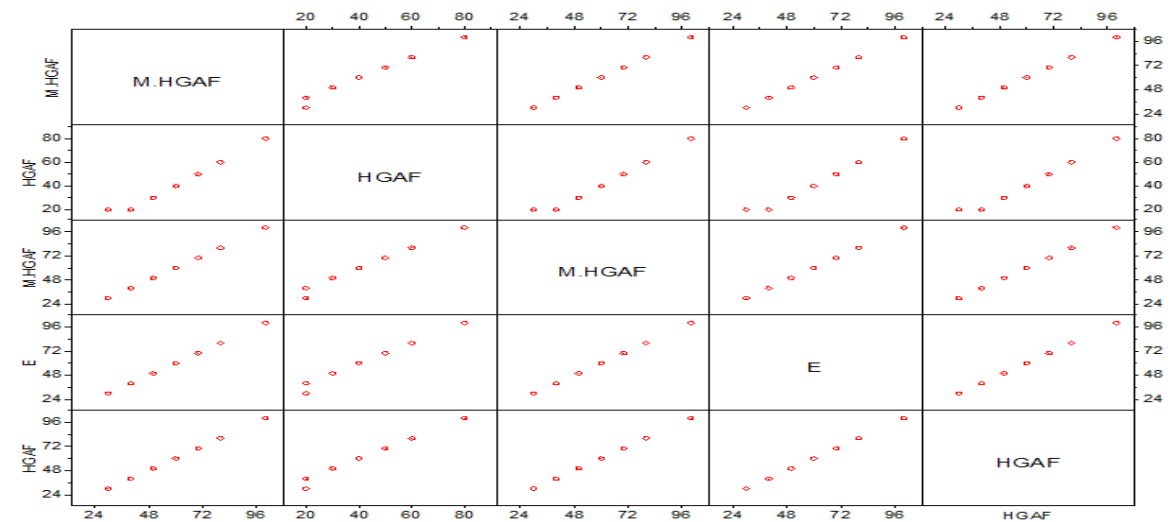

Fig. 8. Communication system in different number of grids

Figure 8 show the different number of gird where the two algorithms communicate with different girds in the network. Figure 9 shows the coverage rate of the two techniques which are used in the network. 


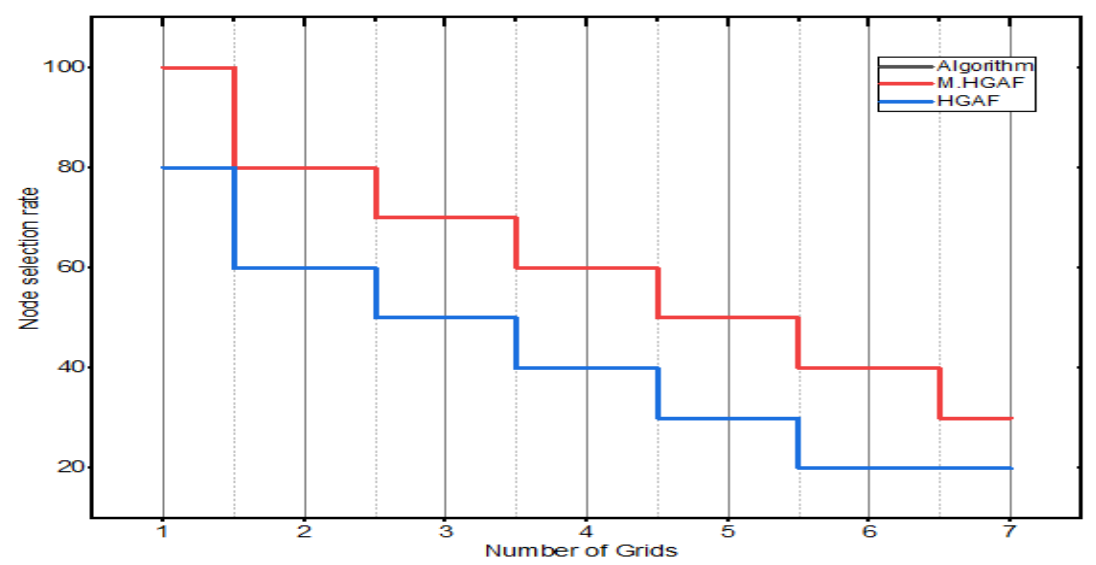

Fig. 9. Both technique coverage rates

Figure 9 shows the coverage ratio rate between the two techniques according to the result the proposed technique has more coverage rate as compared to other techniques. Figure 10 shows the connection system.

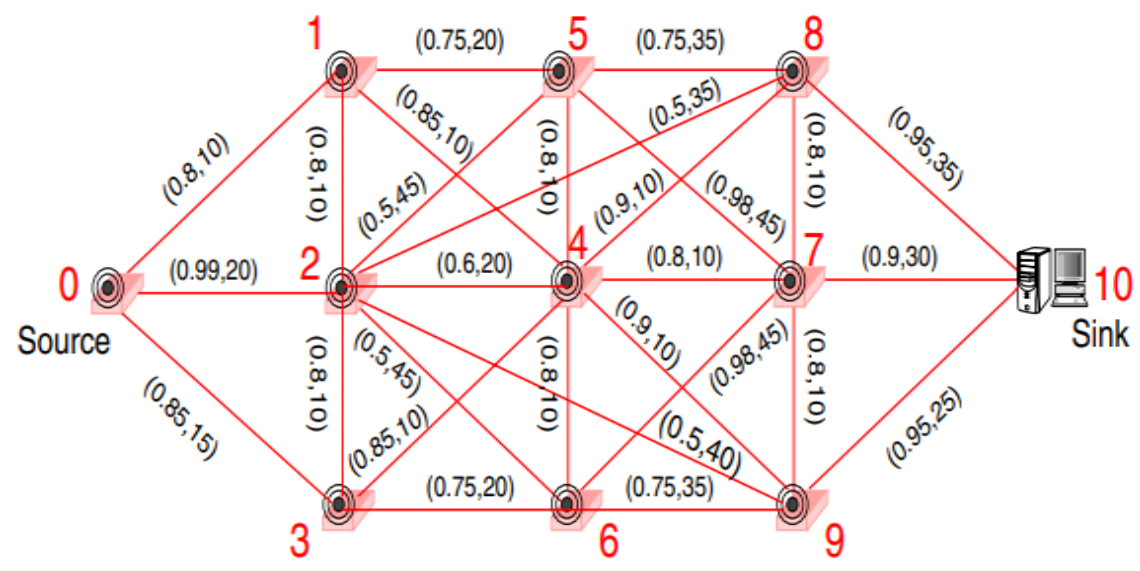

Fig. 10.Connection steps of the different grid in the network

Figures 9 and 10 show the result of path selection in different grids and taking a different parameter. As we mention in the proposed technique sub cell and cell position are change in the grid which improves selection methods and automatically improves the network lifetime. Other results of the network are presented in table 3. 
Table 3. Performance of M.HGAF and HGAF technique

\begin{tabular}{|l|l|l|l|l|}
\hline \multicolumn{1}{|c|}{ G } & \multicolumn{1}{c|}{ G3 } & \multicolumn{1}{c|}{ G4 } & \multicolumn{1}{c|}{ G5 } & \multicolumn{1}{c|}{ G7 } \\
\hline HGAF(3) & 1.6205103 & 604.7200 & 118.9831 & 541.9279 \\
\hline M.HGAF (3) & 0.8718103 & 480.9100 & 65.5373 & 500.2730 \\
\hline HGAF (5) & 1.7101103 & 552.2700 & 97.6902 & 512.4477 \\
\hline M.HGAF (5) & 1.3365103 & 492.8100 & 73.2222 & 494.2294 \\
\hline HGAF (6) & 2.1035103 & 487.2200 & 108.5090 & 493.2639 \\
\hline M.HGAF (6) & 1.7860103 & 461.8600 & 94.2613 & 485.1999 \\
\hline HGAF (7) & 2.0804103 & 388.2600 & 127.3176 & 468.0551 \\
\hline M.HGAF (7) & 2.0746103 & 387.2700 & 121.8136 & 466.1467 \\
\hline
\end{tabular}

Table 3 present the result of different simulation process it consists of four experiments first experiment $3 \times 3$ grid number used second we increase the number up to 5 $\times 5$ and the last one $7 \times 7$ used but the number of nodes are fixed in each simulation and was 1000 nodes. Based on the result it seems that the change of cell in the grid improves the number of the selection process and communication methods are increasing. Due to these increases, it reduces energy consumption and improves the network lifetime. Result shows that in each case the proposed technique result is better than the first one. The energy levels present in figure 11.

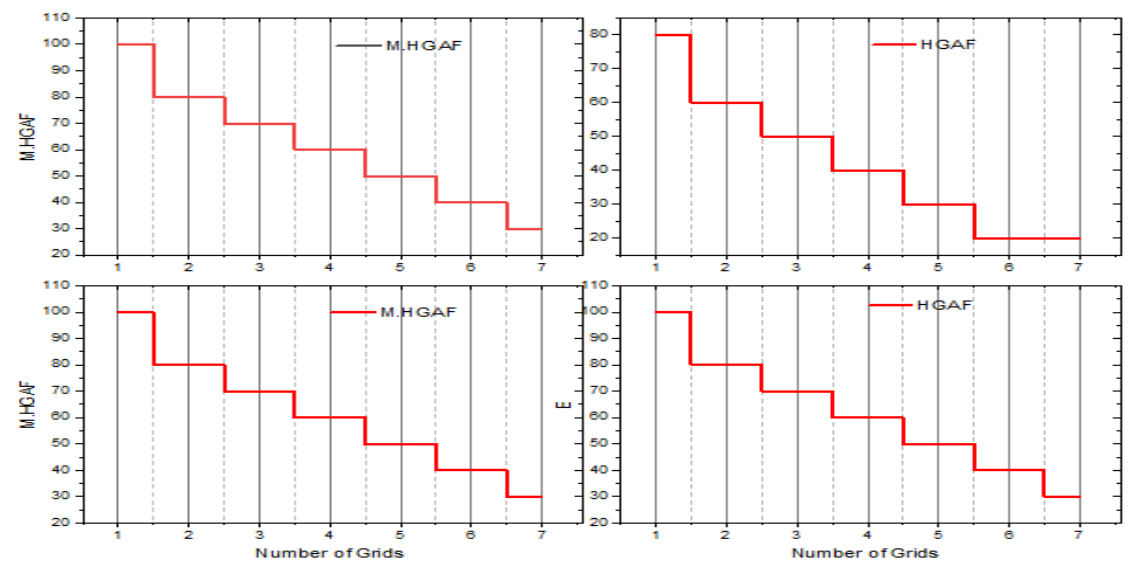

Fig. 11.Energy level of network

Figure 11 show the network life or energy consumption, two different scenarios presented with different parameter and data are mention. Due to sub-cell and cell selection methods, the proper node selection also improves the network energy level. Figure 12 present the reached data to the destination of the network. 


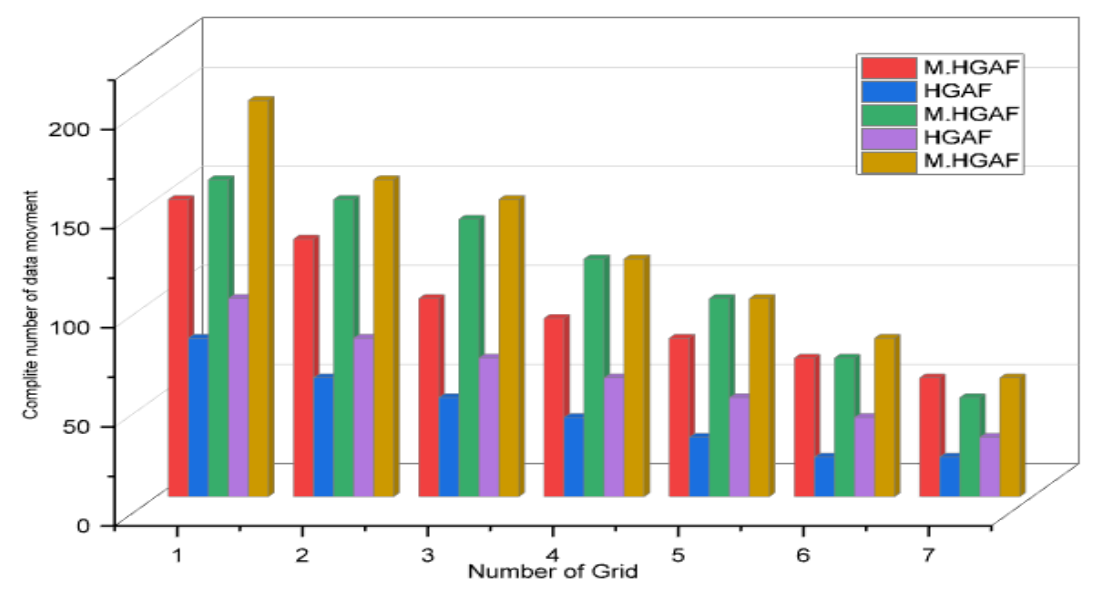

Fig. 12.Data selection in sub cell

Figure 12 present the complete number of data reached the destination using a different number of the grid, the proposed technique sent more number of data to the destination node or sink node. Figure 13 shows the loss number of data during the network execution.

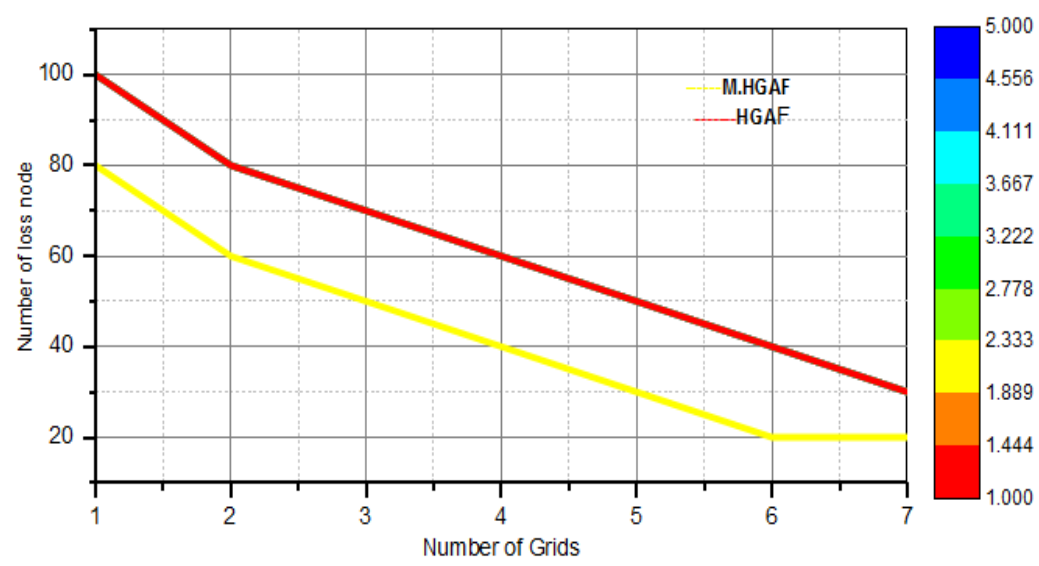

Fig. 13.Present the data loss ratio

Based on the result the data selection methods both cells in grid and sub cell in the grid are used it means which node has a high energy level it selected it. The second main result of the proposed technique is that a fewer number of nodes used to affect the energy level and improve the network life. As mention in figure 13 fewer data loss during the network execution. 


\section{Conclusion}

In this article, we have proposed a new technique for improving the lifetime of the network where we have proposed the modified HGAF protocol in the WSN. The proposed technique is an improved design based on the basic HGAF approach, based on location in a grid cell and sub cell. Our proposed technique selects the path in the cell and sub-cells of the grid and uses an energy-based coordination system for the active node. The MHGAF technique was evaluated based on two scenarios; where we set the number of sensor nodes to 100 , and we used a fixed $3 \times 3$ grid number. While the second scenario consists of a variable grid number from $3 \times 3$ to $9 \times 9$ with several fixed nodes equal to 1000 nodes. Based on the result, our proposed techniques extended the network life and improved the energy life of the sensors, with fewer dead nodes throughout the network run. In the future, we are interested in sensitivity analysis and network connectivity in random nodes and distribution systems.

\section{$7 \quad$ Reference}

[1] Baseer, S., \& Umar, S. (2016, August). Role of cooperation in energy minimization in visual sensor network. In 2016 Sixth International Conference on Innovative Computing Technology (INTECH) (pp. 447-452). IEEE. https://doi.org/10.1109/intech.2016. $\underline{7845026}$

[2] Chiang, M. H., \& Byrd, G. T. (2008, June). Neighborhood-aware density control in wireless sensor networks. In 2008 IEEE International Conference on Sensor Networks, Ubiquitous, and Trustworthy Computing (sutc 2008) (pp. 122-129). IEEE. https://doi.org/10. $1109 /$ sutc. 2008.44

[3] Aznaoui, H., Raghay, S., \& Aziz, L. (2016). New smart nodes distribution using Kmeans approach to enhance routing in WSN. Indian Journal of Science and Technology, 9(46), 2s2. https://doi.org/10.17485/ijst/2016/v9i46/106908

[4] Aznaoui, H., Raghay, S., \& Aziz, L. (2016). Location-based routing protocols gaf and its enhanced versions in wireless sensor network a survey. International Journal of Computer Science and Information Security, 14(6), 497.

[5] Wan, Q., \& Wang, Y. (2018). Exploration of Wireless Sensor Network Based on Cloud Computing. International Journal of Online and Biomedical Engineering (iJOE), 14(11), 16-27. https://doi.org/10.3991/ijoe.v14i11.9501

[6] Xu, J. (2018). A New Method for Reliability Evaluation of Wireless Sensor Networks Based on Fuzzy Neural Network. International Journal of Online and Biomedical Engineering (iJOE), 14(10), 180-192. https://doi.org/10.3991/ijoe.v14i10.9315

[7] Gao, Y. (2018). Implementation of an Intelligent Library System Based on WSN and RFID. International Journal of Online and Biomedical Engineering (iJOE), 14(05), 211224. https://doi.org/10.3991/ijoe.v14i05.8601

[8] Fang, J. (2019). Clustering and Path Planning for Wireless Sensor Networks Based on Improved Ant Colony Algorithm. International Journal of Online and Biomedical Engineering (iJOE), 15(01), 129-142. https://doi.org/10.3991/ijoe.v15i01.9784

[9] Wan, Q., Weng, M. J., \& Liu, S. (2019). Optimization of wireless sensor networks based on differential evolution algorithm. International Journal of Online and Biomedical Engineering (iJOE), 15(01), 183-195. https://doi.org/10.3991/ijoe.v15i01.9786 
[10] Ghaderi, M. R., Vakili, V. T., \& Sheikhan, M. (2019). FGAF-CDG: fuzzy geographic routing protocol based on compressive data gathering in wireless sensor networks. Journal of Ambient Intelligence and Humanized Computing, 1-23. https://doi.org/10.1007/s1265 2-019-01314-1

[11] Lavanya, N., \& Shankar, T. (2019). Energy Efficient Cluster Head Selection using Hybrid Squirrel Harmony Search Algorithm in WSN. Energy, 10(12). https://doi.org/10.14569/ ijacsa.2019.0101265

[12] Aznaoui, H., Raghay, S., Ouakrim, Y., \& Aziz, L. (2019). A Heuristic Algorithm of Cooperative Agents Communication for Enhanced GAF Routing Protocol in WSNs. Wireless Communications and Mobile Computing, 2019. https://doi.org/10.1155/2019/4947610

[13] Ouhame, S., Hadi, Y., \& Arifullah, A. (2020). A Hybrid Grey Wolf Optimizer and Artificial Bee Colony Algorithm Used for Improvement in Resource Allocation System for Cloud Technology. https://doi.org/10.3991/ijoe.v16i14.16623

[14] Ullah, A., \& Nawi, N. M. (2020). Enhancing the dynamic load balancing technique for cloud computing using HBATAABC algorithm. International Journal of Modeling, Simulation, and Scientific Computing, 11(05), 2050041. https://doi.org/10.1142/s179396 2320500415

[15] Fotohi, R., Firoozi Bari, S., \& Yusefi, M. (2020). Securing wireless sensor networks against denial-of-sleep attacks using RSA cryptography algorithm and interlock protocol. International Journal of Communication Systems, 33(4), e4234. https://doi.org/10.10 02/dac. 4234

[16] Han, F., Abdelaziz, I. I. M., Liu, X., \& Ghazali, K. H. (2020). A Hybrid Range-Free Algorithm Using Dynamic Communication Range for Wireless Sensor Networks. International Journal of Online \& Biomedical Engineering, 16(8). https://doi.org/10.3991/ijoe.v16 $\underline{\mathrm{i} 08.14379}$

[17] Sinde, R., Begum, F., Njau, K., \& Kaijage, S. (2020). Refining Network Lifetime of Wireless Sensor Network Using Energy-Efficient Clustering and DRL-Based Sleep Scheduling. Sensors, 20(5), 1540. https://doi.org/10.3390/s20051540

[18] Srivastava, V., Tripathi, S., \& Singh, K. (2020). Energy efficient optimized rate-based congestion control routing in wireless sensor network. Journal of Ambient Intelligence and Humanized Computing, 11(3), 1325-1338. https://doi.org/10.1007/s12652-019-0144 9-1

[19] Ullah, A., Nawi, N. M., Aamir, M., Shazad, A., \& Faisal, S. N. An Improved Multi-layer Cooperation Routing in Visual Sensor Network for Energy Minimization. https://doi.org/ 10.18517/ijaseit.9.2.2957

[20] Abd Aziz, A., Sekercioglu, Y. A., Fitzpatrick, P., \& Ivanovich, M. (2012). A survey on distributed topology control techniques for extending the lifetime of battery powered wireless sensor networks. IEEE communications surveys \& tutorials, 15(1), 121-144. https://doi.org/10.1109/surv.2012.031612.00124

[21] Abella, C. S., Bonina, S., Cucuccio, A., D’Angelo, S., Giustolisi, G., Grasso, A. D., \& Pennisi, S. (2019). Autonomous energy-efficient wireless sensor network platform for home/office automation. IEEE Sensors Journal, 19(9), 3501-3512. https://doi.org/10. 1109/jsen.2019.2892604

[22] Ali, B., Mahmood, T., Mirza, M. A., Memon, S., Rashid, M., \& Ajebesone, E. F. Study and Analysis of Delay Sensitive and Energy Efficient Routing Approach. system, 26, 27.

[23] Anwar, Arifa, and D. Sridharan. "A survey on routing protocols for wireless sensor networks in various environments." International Journal of Computer Applications 112, no. 5 (2015). 
[24] Carminati, M., Turolla, A., Mezzera, L., Di Mauro, M., Tizzoni, M., Pani, G., ... \& Antonelli, M. (2020). A self-powered wireless water quality sensing network enabling smart monitoring of biological and chemical stability in supply systems. Sensors, 20(4), 1125. https://doi.org/10.3390/s20041125

[25] Chan, Edward, and Song Han. "Energy efficient residual energy monitoring in wireless sensor networks." International Journal of Distributed Sensor Networks 5, no. 6 (2009): 748-770. https://doi.org/10.1080/15501320902876055

[26] Saleem, Muhammad, Israr Ullah, and Muddassar Farooq. "BeeSensor: An energy-efficient and scalable routing protocol for wireless sensor networks." Information Sciences 200 (2012): 38-56. https://doi.org/10.1016/j.ins.2012.02.024

[27] Sendra Compte, Sandra, Jaime Lloret, Miguel García Pineda, and José Francisco Toledo Alarcón. "Power saving and energy optimization techniques for wireless sensor networks." Journal of communications 6, no. 6 (2011): 439-459. https://doi.org/10.4304/jcm.6.6 $.439-459$

[28] Shabbir, Noman, and Syed Rizwan Hassan. "Routing protocols for wireless sensor networks (WSNs)." Wireless Sensor Networks-Insights and Innovations (2017). https://doi.org/10.5772/intechopen.70208

[29] ul Hassan, Mahmood, Amin Al Awady, Khalid Mahmood, Shahzad Ali, and Muhammad Kashif Saeed. "Node Relocation Techniques for Wireless Sensor Networks: A Short Survey." https://doi.org/10.14569/ijacsa.2019.0101145

[30] Ullah, A., Nawi, N. M., Sutoyo, E., Shazad, A., Khan, S. N., \& Aamir, M. (2018). Search Engine Optimization Algorithms for Page Ranking: Comparative Study. International Journal of Integrated Engineering, 10(6).

[31] Aznaoui, H., Raghay, S., Aziz, L., Benloghfyry, A., \& Duvallet, C. (2018). Efficient Weighted GAF routing protocol using data aggregation in WSN.

\section{Authors}

Hanane Aznaoui working as scholar at Cadi Ayyad University, Faculty of Sciences and Technology, Laboratoire de Mathématiques Appliquées et Informatique (LAMAI), Marrakech, Morocco. Email : $\underline{\text { H.aznaoui@gmail.com }}$

Dr Said Raghay working as Prof Cadi Ayyad University, Faculty of Sciences and Technology, Laboratoire de Mathématiques Appliquées et Informatique (LAMAI), Marrakech, Morocco.

Arif Ullah working as scholar Faculty of Computer Science and Information Technology Universiti Tun Hussein Onn Malaysia, Malaysia. Email: Arifullahms88@ gmail.com

Mubashir Hayat Khan working as scholar Faculty of Electrical and Electronic Engineering, Universiti Tun Hussein Onn Malaysia, Malaysia

Article submitted 2020-08-13. Resubmitted 2021-01-20. Final acceptance 2021-03-07. Final version published as submitted by the authors. 